\section{BMJ Open Respiratory Research}

\title{
Early extracellular matrix changes are associated with later development of bronchiolitis obliterans syndrome after lung transplantation
}

\author{
Catharina Müller, ${ }^{1}$ Annika Andersson-Sjöland, ${ }^{1}$ Hans Henrik Schultz, ${ }^{2}$ \\ Leif T Eriksson, ${ }^{1,3}$ Claus B Andersen, ${ }^{4}$ Martin Iversen, ${ }^{2}$ \\ Gunilla Westergren-Thorsson ${ }^{1}$
}

To cite: Müller C, AnderssonSjöland A, Schultz $\mathrm{HH}$, et al. Early extracellular matrix changes are associated with later development of bronchiolitis obliterans syndrome after lung transplantation. BMJ Open Resp Res 2017;4:e000177. doi:10.1136/bmjresp-2016000177

- Additional material is published online only. To view please visit the journal online (http://dx.doi.org/10. 1136/bmjresp-2016-000177)

Received 16 December 2016 Revised 3 February 2017 Accepted 6 February 2017

For numbered affiliations see end of article.

Correspondence to Dr Catharina Müller; catharina.muller@med.Iu.se

\section{ABSTRACT}

Background: Chronic lung allograft dysfunction in the form of bronchiolitis obliterans syndrome (BOS) is the main cause of death beyond 1-year post-lung transplantation. The disease-initiating triggers as well as the molecular changes leading to fibrotic alterations in the transplanted lung are largely unknown. The aim of this study was to identify potential early changes in the extracellular matrix (ECM) in different compartments of the transplanted lung prior to the development of BOS.

Methods: Transbronchial biopsies from a cohort of 58 lung transplantation patients at the Copenhagen University hospital between 2005 and 2006, with or without development of BOS in a 5-year follow-up, were obtained 3 and 12 months after transplantation. Biopsies were assessed for total collagen, collagen type IV and biglycan in the alveolar and small airway compartments using Masson's Trichrome staining and immunohistochemistry.

Results: A time-specific and compartment-specific pattern of ECM changes was detected. Alveolar total collagen $(p=0.0190)$ and small airway biglycan $(p=0.0199)$ increased between 3 and 12 months after transplantation in patients developing BOS, while collagen type IV ( $p=0.0124)$ increased in patients without BOS. Patients with early-onset BOS mirrored this increase. Patients developing grade 3 BOS showed distinct ECM changes already at 3 months. Patients with BOS with treated acute rejections displayed reduced alveolar total collagen $(p=0.0501)$ and small airway biglycan ( $p=0.0485)$ at 3 months.

Conclusions: Patients with future BOS displayed distinct ECM changes compared with patients without BOS. Our data indicate an involvement of alveolar and small airway compartments in post-transplantation changes in the development of BOS.

\section{INTRODUCTION}

Lung transplantation (ltx) is a life-saving treatment option for patients with severe chronic pulmonary diseases. ${ }^{1}$ Unfortunately, about

\section{KEY MESSAGES}

Do changes in the extracellular matrix precede the development of bronchiolitis obliterans syndrome after lung transplantation?

- Total collagen, collagen type IV and biglycan show compartment-specific changes after transplantation which distinguish patients who do or do not develop bronchiolitis obliterans syndrome.

- The identification of time-specific and compartment-specific molecular changes after transplantation is a precondition for a better understanding of disease development in the future.

$50 \%$ of the patients develop chronic lung allograft dysfunction (CLAD) within 5 years after transplantation, leading to decreased lung function and organ failure. ${ }^{2}$ CLAD is defined by a persistent decline in forced expiratory volume in $1 \mathrm{~s}\left(\mathrm{FEV}_{1}\right)$ below $80 \%$ of baseline, while excluding other possible reasons. ${ }^{3}$ Two subtypes are known: the more common obstructive phenotype called bronchiolitis obliterans syndrome (BOS) and a recently identified restrictive phenotype. ${ }^{4-6}$ The common theory is that a damage-inducing event during or after transplantation triggers an epithelial repair response with activation of inflammatory and non-inflammatory mediators leading to fibrosis. ${ }^{7}$ Risk factors for CLAD are recognised with acute cellular rejection episodes remaining the most important one. ${ }^{6}$ However, the damaging insult and the concrete molecular changes resulting in fibrosis remain unknown. After acute cellular rejection has resolved, transbronchial biopsies seem normal in routine investigation with apparently no residual damage left.

The present study focuses on BOS. BOS severity is graded according to the $\mathrm{FEV}_{1}$ decrease (BOS 1: 66-80\%, BOS 2: 51-65\%, 
BOS 3: $<50 \% \mathrm{FEV}_{1}$ of baseline). ${ }^{6}$ In biopsies, obliterative bronchiolitis lesions can be found in small airways; however, these are focal in nature and easily missed. There is a strong need to investigate molecular changes leading to BOS with the aim to improve diagnostic tools and to understand pathological mechanisms, for example, by gene expression profiling and evaluation of subsets of immune-modulating cells in bronchoalveolar lavage. ${ }^{9-12}$

We hypothesise that until now unknown triggers of BOS lead to changes in the extracellular matrix (ECM), possibly in different parts of the transplanted lung. These changes might be signs of ongoing remodelling processes that, at a later stage, lead to increased susceptibility to BOS.

We analysed the content of total collagen, collagen type IV (col IV), and biglycan in transbronchial biopsies of 58 lung transplanted patients with or without BOS, both at 3 and 12 months post-ltx. We assessed both alveolar parenchyma and small airways. Changes in small airways are well described, but there is evidence of involvement of alveolar tissue in BOS as well. ${ }^{13} 14$

Collagens are believed to be among the most highly deposited proteins during fibrosis. ${ }^{15} \mathrm{Col}$ IV is present in basement membranes (BM) and we focused on the predominant isoform ( $\alpha$-chain 1/1/2-trimer) in the adult lung. ${ }^{16-18}$ Changes in the BM might be directly correlated to epithelial damage. Biglycan is a proteoglycan with complex functions in tissue homoeostasis due to its capacity to sequester soluble factors. ${ }^{19}{ }^{20}$ Increased biglycan was found in central airways and alveolar parenchyma of patients with uncontrolled asthma. ${ }^{21}$ Furthermore, fibroblasts isolated from patients who developed BOS were shown to be more responsive to TGF- $\beta 1$-induced biglycan expression compared with stable patients. ${ }^{22}$

The systematic quantification of these proteins revealed a time-specific and localisation-specific pattern of ECM changes, which differed between patients who did or did not develop BOS.

\section{MATERIALS AND METHODS \\ Study population}

All patients undergoing ltx at Copenhagen University Hospital (Rigshospitalet, Denmark) in 2005 and 2006 were included in this study $(n=60)$. Therefore, this patient set is likely to be an unbiased cohort of lung transplant recipients. This material has been described previously with an emphasis on regulatory $\mathrm{T}$ cells. ${ }^{12}$ Patient characteristics with a focus on BOS and acute rejections are summarised in table 1. Online supplementary figure S1 depicts the two subpopulations of patients with early and late onset of BOS.

Two patients died shortly after transplantation. Fifty-eight patients entered the surveillance follow-up programme with regular transbronchial biopsies and lung function measurements. No major bleedings or
Table 1 Recipient characteristics

\begin{tabular}{ll}
\hline Mean recipient age (years) & 42 (range 14-66) \\
Recipient gender & 25 women, 33 men \\
Transplant type (number of patients) & \\
Single lung & 26 \\
Double lung & 31 \\
Heart lung & 1 \\
Number of patients with n numbers of treated acute \\
rejection episodes (within 3/12 months post-Itx) \\
$\mathrm{n}=0$ & $31 / 28$ \\
$\mathrm{n}=1$ & $10 / 14$ \\
$\mathrm{n}=2$ & $14 / 11$ \\
$\mathrm{n}=3$ & $2 / 5$ \\
Number of patients developing BOS & 28 of 58 \\
Early onset (0.4-1.2 years post-Itx) & 15 \\
Late onset (2.3-4.4 years post-Itx) & 13 \\
Number of patients (early/late onset) & with maximal BOS \\
grade of & \\
Grade 1 & $1 / 6$ \\
Grade 2 & $1 / 5$ \\
Grade 3 & $13 / 2$ \\
\hline
\end{tabular}

*Within 5 years post-Itx follow-up.

BOS, bronchiolitis obliterans syndrome; Itx, lung transplantation.

pneumothoraxes occurred. Details of the immunosuppressive regimen (induction therapy with antithymocyte globulin, maintenance therapy with cyclosporine and azathioprine with prednisolone) have been described previously. ${ }^{12}{ }^{23}$ Patients were treated with azithromycin when diagnosed with BOS.

In the frame of this study, patients were followed for 5 years and assessed whether they did or did not develop BOS. There was no significant difference in the ischaemia time between the two groups. Per-protocol surveillance biopsies from 3 and 12 months were analysed retrospectively. Retrospective analysis does not require ethical committee approval but the study was approved by the Danish Data Protection Agency. Biopsies were scored according to current ISHLT criteria for the diagnosis of lung rejection by an experienced pathologist (CBA). ${ }^{3}$ Only treated acute cellular rejection episodes were considered in this study (grade A2 or higher, treatment with methylprednisolone).

Biopsies with an insufficient amount of alveolar tissue or small airway areas, obviously missing staining due to biopsy preparation artefacts or with heavily destroyed tissue morphology, were excluded.

\section{Masson's Trichrome staining}

Masson's Trichrome staining to assess total collagen content was performed on $4 \mu \mathrm{m}$ sections from formalinfixed, paraffin-embedded biopsies as a routine procedure at the Department of Pathology, Copenhagen University Hospital, Denmark.

\section{Immunohistochemistry}

An immunohistochemistry (IHC) double-staining procedure visualised col IV and biglycan on $4 \mu \mathrm{m}$ sections 
from formalin-fixed, paraffin-embedded biopsies using the EnVision Doublestain System (K5361, Dako, Glostrup, Denmark) according to the manufacturer's instructions. Single-staining tests for each antibody showed that the locations for the two proteins were not overlapping, thereby enabling quantification of each target in the double staining procedure. Antibodies were assessed for specificity and optimal antigen retrieval methods. ${ }^{21}$ Antigen retrieval was performed with $0.2 \%$ bovine testicular hyaluronidase (Sigma type I-S, buffer $20 \mathrm{mM}$ sodium acetate, $150 \mathrm{mM}$ sodium chloride, $\mathrm{pH} 5.2$ ) for $30 \mathrm{~min}$ and subsequently with $20 \mu \mathrm{g} / \mathrm{mL}$ protease XXIV in PBS (Subtilisin, Carlsberg, Sigma) for $20 \mathrm{~min}$ (both room temperature). Primary antibodies against col IV $\alpha 1$ chain (Abcam 6586, concentration $1 \mathrm{mg} / \mathrm{mL}$, used at 1:4000) and biglycan (Atlas antibodies, Uppsala, Sweden, HPA003157, concentration $0.1 \mathrm{mg} / \mathrm{mL}$, used at 1:1750) were incubated for 1 hour at room temperature. Secondary antibodies produced brown (col IV) or red (biglycan) precipitates. Sections were counterstained with Mayer's haematoxylin for visualisation of nuclei.

\section{Tissue analysis and quantification}

Sections were scanned using the ScanScope slide scanner (Aperio, Vista, California, USA). Masson's Trichrome staining and extent of immunoreactivity against col IV and biglycan were quantified using the histology programme Visiomorph (Visiopharm, Hoersholm, Denmark). After defining the categories (positive staining, unstained tissue, background), the programme quantified the respective areas, thus allowing exclusion of the background so that air spaces were excluded from analysis. Satisfactory discrimination of positive col IV (brown) and biglycan (red) staining was checked in all samples. The different compartments were delineated manually and analysed separately.

Alveolar tissue: Included were clearly discernible alveolar regions. Other structures (cartilage, muscle, vessels larger than $\sim 75 \mu \mathrm{m}$ and cell accumulations in the alveoli) were carefully excluded. The percentage of positively stained area was obtained by dividing the stained area by the total tissue area, multiplied by 100 .

Small airways: Small airways were defined by the absence of cartilage and submucosal glands. The analysed area was defined as the area from below the epithelial layer (including the $\mathrm{BM}$ ) to (but not including) the airway smooth muscle. Only airways where muscle was visible, or where extrapolation between streaks of muscles was possible, were analysed. Epithelial cells or an intact BM had to be visible to ensure complete areas. Quantification of positive staining was performed by relating the area of positive staining to the length of the epithelium.

All tissue analyses as well as IHC were performed in a blinded fashion by one of the investigators $(\mathrm{CM})$.

\section{Statistical analysis}

Data analyses were performed using GraphPad Prism V.6.05 (GraphPad Software, San Diego, California, USA) and are presented as scatter dot plots with median values. The non-parametric Wilcoxon matched-pairs signed-rank test was used for paired data; the nonparametric Mann-Whitney test was used for unpaired analysis as indicated in the figures.

\section{RESULTS}

\section{General distribution of total collagen, col IV and biglycan}

Masson's Trichrome staining visualised collagen in the airways, often clearly depicting the reticular BM, but also reaching deeper (see online supplementary figure S2A). It stained interstitial ECM (see online supplementary figure S2C) as well as the vascular adventitia (see online supplementary figure S2B,C). Alveolar septa stained variably, from thick ECM bundles to fine fibrillar structures (see online supplementary figure S2B and figure $1 \mathrm{~A}^{\prime}-\mathrm{A} "$ ").

Col IV was detected in the BM of alveolar septa (see online supplementary figure S2F and figure 1B'-B"'), airways (see online supplementary figure S2E and figure 1C'-C'”), capillaries (see online supplementary figure S2F), and muscle fibres in the airway musculature and in big and small vessels (see online supplementary figure S2F,G).

Biglycan was detected in subepithelial regions of airways, often accumulating close to the BM (see online supplementary figure S2E and figure $1 C^{\prime}-C^{\prime \prime}$ ), in the adventitia of larger vessels (see online supplementary figure S2G) and around small vessels (see online supplementary figure S2F). Alveolar septa showed biglycan in patchy or fine structures (see online supplementary figure S2F and figure 1B'-B"'). Staining of the pericellular matrix of cartilage confirmed the specificity of the biglycan antibody (see online supplementary figure S2D). ${ }^{24}$ Omitting primary antibodies resulted in total absence of staining, thus confirming the specificity of the secondary antibodies (see online supplementary figure $\mathrm{S} 2 \mathrm{H}$ ).

\section{Compartment-specific increase in total collagen, col IV and biglycan between 3 and 12 months after Itx}

On the group level, total collagen and col IV increased in the alveolar compartment, but not in the small airways, between 3 and 12 months post-ltx (figure 1A, B; Wilcoxon matched-pairs test, $\mathrm{p}=0.0247$ and $\mathrm{p}=0.0024$, respectively). In contrast, biglycan increased in the small airways, but not in the alveolar compartment (figure 1C; Mann-Whitney test, $\mathrm{p}=0.0475$ ). Representative pictures are shown in figure 1, while enlarged figures are shown in online supplementary figures S3A-C.

\section{Patients with BOS displayed compartment-specific post-Itx changes compared with patients without BOS}

On the group level, patients with BOS showed increased alveolar total collagen between 3 and 12 months (figure 2A; Wilcoxon matched-pairs test, $\mathrm{p}=0.019$ ), while patients without BOS did not (figure 2A'; 


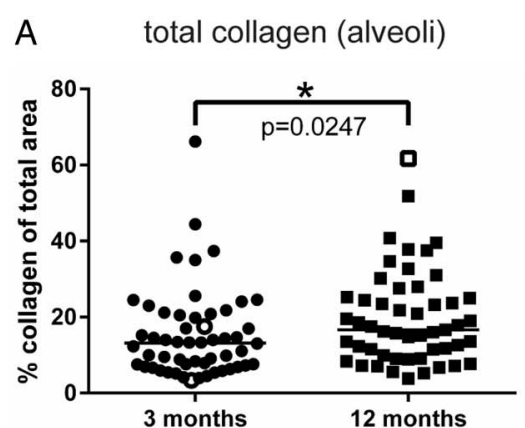

Masson's Trichrome (alveoli)

total collagen (blue)

nuclei (dark red)

other structures (light red)
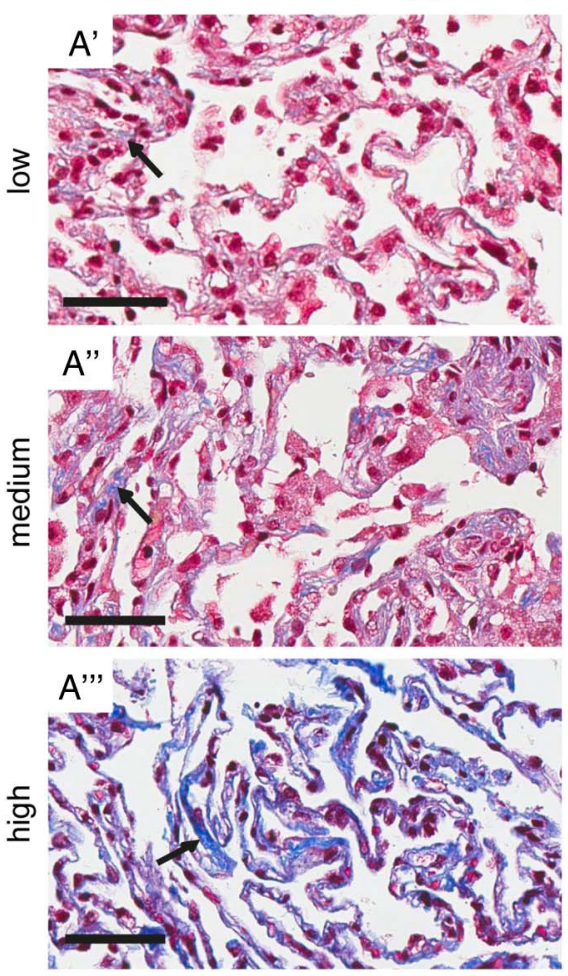

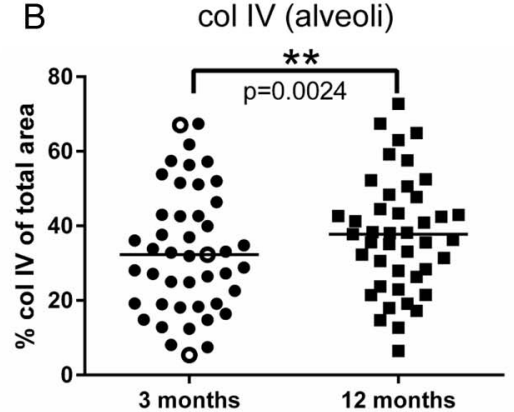

Double-IHC col IV-biglycan (alveoli)

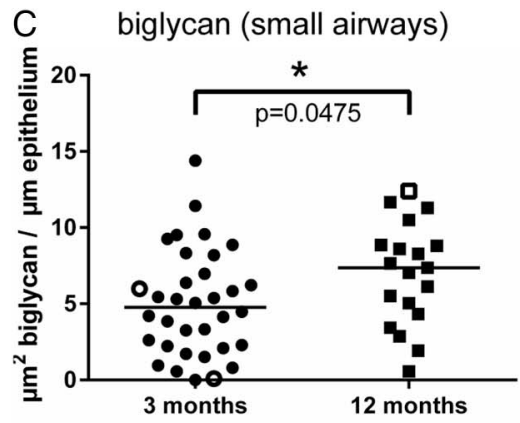

Double-IHC col IV-biglycan (small airways) $\square$ col IV (brown)

$\square$ biglycan (red)

$\square$ nuclei (lilac)
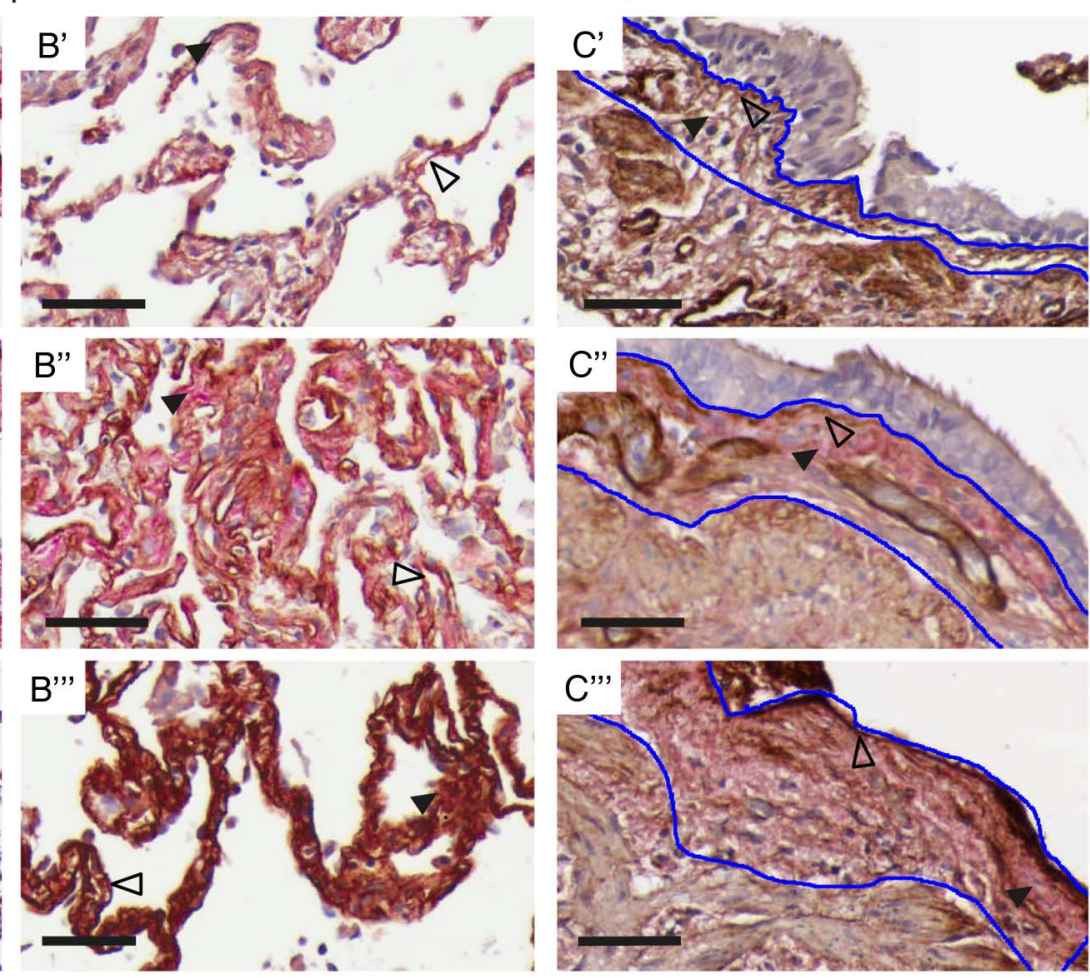

Figure 1 ECM changes between 3 and 12 months post-transplantation in different pulmonary compartments. Total collagen (A) and col IV (B) were increased in the alveoli, and biglycan (C) was increased in the small airways ( 3 vs 12 months, total collagen and col IV: Wilcoxon matched-pairs test, biglycan: Mann-Whitney test). Data are shown as scatter dot plots with median values. Open symbols in $\mathrm{A}-\mathrm{C}$ indicate the individual biopsies shown in the pictures. Representative pictures of biopsies with low, medium and high staining intensity for alveolar total collagen (A'-A'”), alveolar (B'-B'") and small airway biglycan/col IV (C'-C'”). Scale bar $=50 \mu \mathrm{m}$, Masson's Trichrome total collagen: blue $(\rightarrow)$, col IV: brown $(\Delta)$, biglycan: red $(\rightarrow)$. Blue lines indicate regions of interest for quantification of the staining in the small airways. Col IV, collagen type IV; ECM, extracellular matrix; IHC, immunohistochemistry.

$\mathrm{p}=0.784)$. The same pattern was observed for biglycan, however, only in the small airways (figure 2C, C'; Mann-Whitney test, $\mathrm{p}=0.0199$ for patients with BOS, $\mathrm{p}=0.8514$ for patients without BOS). On the other hand, patients who did not develop BOS displayed significantly increased alveolar col IV (figure 2B'; Wilcoxon matchedpairs test, $\mathrm{p}=0.0124$ ) while it only slightly increased in patients developing BOS (figure 2B; $\mathrm{p}=0.0730$ ). No difference could be observed when comparing patients with or without BOS at 3 or 12 months, respectively.

Biopsies from the same patient at 3 and 12 months post-ltx allowed for comparisons of ECM changes over time within each individual (figure 2A", B", C"). Depicted is the difference of total collagen, col IV and biglycan per individual (value at 12 months minus value at 3 months). For small airway biglycan, a distinct 

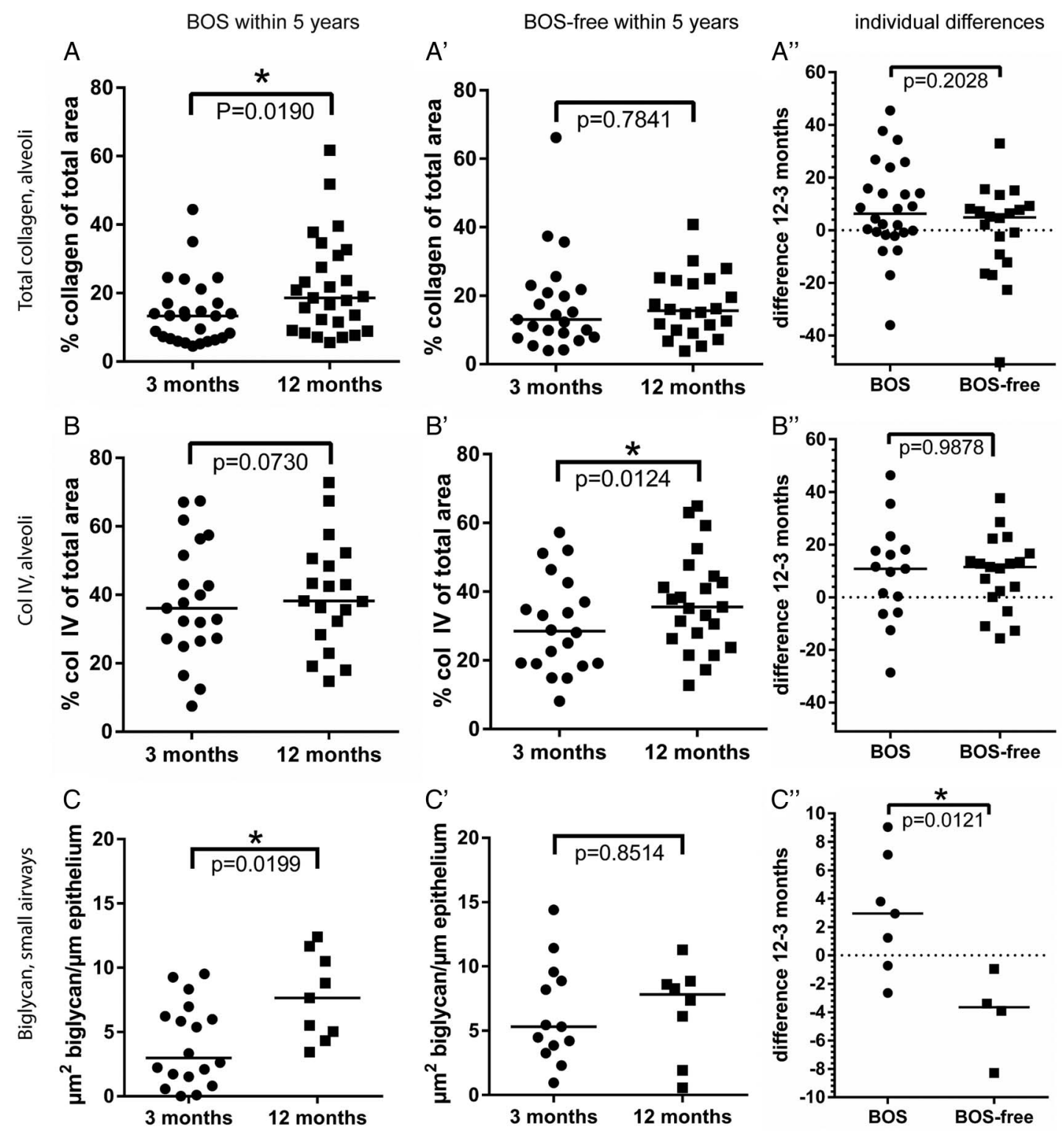

Figure 2 ECM changes between 3 and 12 months post-Itx in patients with or without BOS in the different pulmonary compartments. Changes in total alveolar collagen, alveolar col IV and small airway biglycan between 3 and 12 months post-Itx in patients developing BOS (A, B, C) and patients without BOS (A', B', C') on the group level. Difference in total alveolar collagen (A"), alveolar col IV (B") and small airway biglycan (C") for each individual patient between 3 and 12 months post-ltx. Scatter dot plots are shown with median values. Statistical tests were Wilcoxon matched-pairs test in A, A', B, B' and Mann-Whitney test in A", B", C-C". BOS, bronchiolitis obliterans syndrome; col IV, collagen type IV; ECM, extracellular matrix; Itx, lung transplantation.

individual difference between patients developing BOS and patients without BOS was observed (Mann-Whitney test, $\mathrm{p}=0.0121$; figure $2 \mathrm{C}$ ").

\section{Patients with early-onset BOS showed distinct ECM changes between 3 and 12 months}

In our study, patients with BOS exhibited early-onset BOS (within 1.2 years) and late-onset BOS (2.3 years or later; table 1 and online supplementary figure $\mathrm{S} 1$ ). Thirteen of 15 early-onset patients proceeded to BOS grade 3 within the 5-year follow-up, while 2 of 13 late-onset patients developed BOS grade 3 in the same time frame.
Only patients with early-onset BOS showed increased alveolar total collagen and col IV (figure 3A, B; Wilcoxon matched-pairs test, $\mathrm{p}=0.0327$ and $\mathrm{p}=0.0391$, respectively) and small airway biglycan (figure 3C; Mann-Whitney test, $\mathrm{p}=0.025$ ) between 3 and 12 months post-ltx.

\section{BOS severity and early ECIM changes}

Patients proceeding to BOS grade 3 showed higher content of alveolar total collagen (figure 3D) and small airway col IV (figure 3E) at 3 months post-ltx compared with patients who had a maximum BOS grade of 1 or 2 (Mann-Whitney test, $\mathrm{p}=0.0362$ and $\mathrm{p}=0.0343$, respectively). 

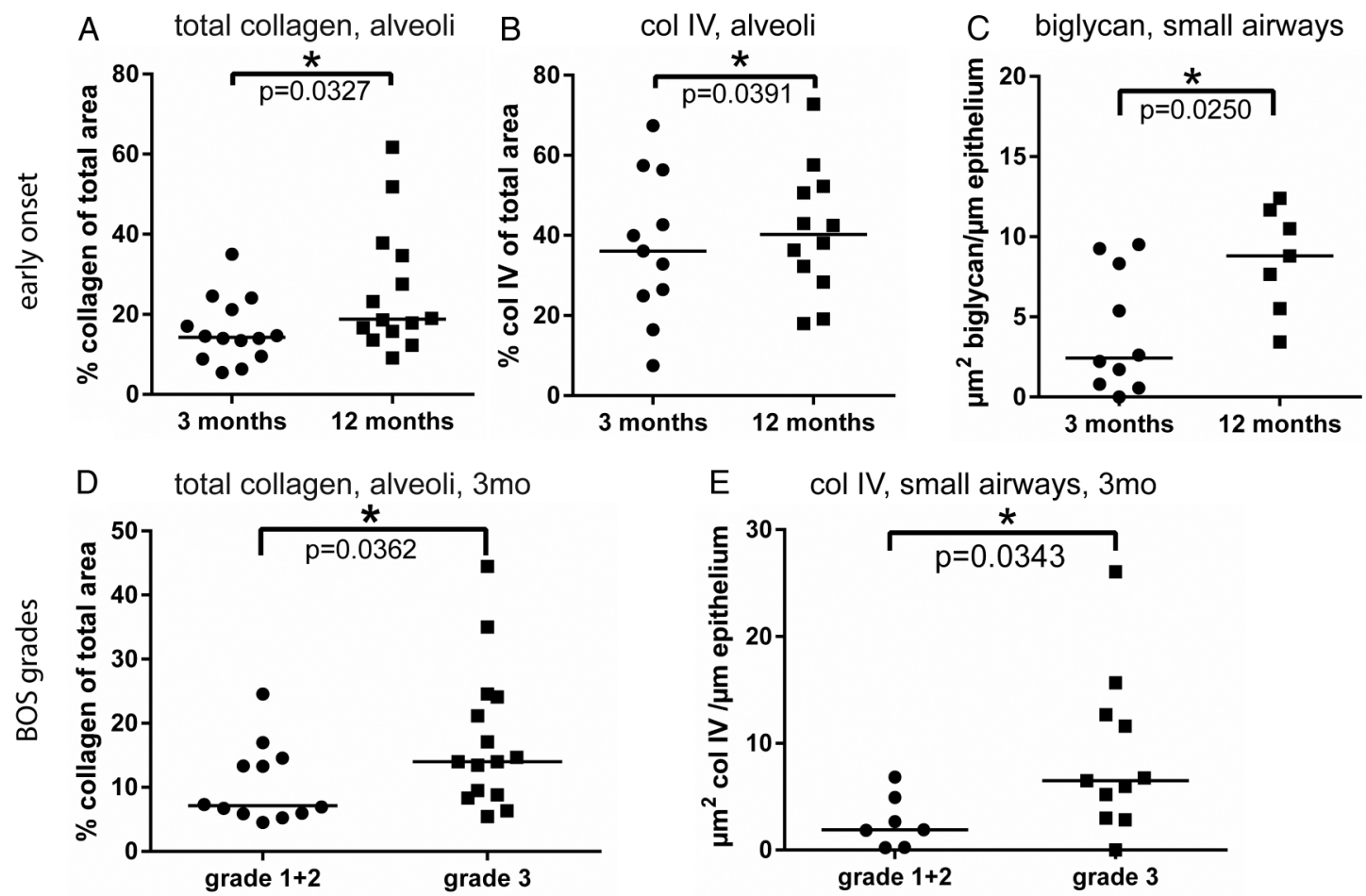

Figure 3 Subgrouping of the patient population according to early-onset/late-onset BOS and maximal BOS grade. Increase in total alveolar collagen (A), alveolar col IV (B) and small airway biglycan (C) in early-onset patients with BOS between 3 and 12 months post-Itx. Patients proceeding to BOS grade 3 within the follow-up showed increased total alveolar collagen (D) and small airway col IV (E) compared with patients with a maximum of BOS grade 1 or 2. Scatter dot plots are shown with median values. Statistical tests were Wilcoxon matched-pairs test in A-B and the Mann-Whitney test in C-E. BOS, bronchiolitis obliterans syndrome; col IV, collagen type IV; Itx, lung transplantation.

\section{Acute rejection episodes, early compartment-specific ECM changes and later development of BOS}

Patients who later developed BOS and suffered from treated acute rejections within 3 months post-ltx showed lower total alveolar collagen (figure 4A) and small airway biglycan (figure 4C) at 3 months post-ltx, compared with patients with BOS who experienced no treated acute rejections (Mann-Whitney test, $\mathrm{p}=0.004$ and $\mathrm{p}=0.0266$, respectively). This was not observed for patients without BOS (figure 4A', C'). When directly comparing the alveolar collagen content and the small airway biglycan content in patients developing BOS compared with patients without BOS (both groups with treated acute rejection within 3 months), this decrease was observed as well (figure 4A", C"; Mann-Whitney test, $\mathrm{p}=0.0501$ and $\mathrm{p}=0.0485$, respectively).

In contrast, a difference in alveolar col IV at 3 months was observed in patients who did not experience treated acute rejection episodes (figure 4B"). Here, patients who developed BOS showed increased alveolar col IV content compared with patients without BOS (Mann-Whitney test, $\mathrm{p}=0.0492$ ). There was no difference when comparing patients with or without treated acute rejections (figure 4B, B').

No differences were observed with regard to total collagen, col IV and biglycan content at 12 months in relation to the number of treated acute rejections until 12 months post-ltx (data not shown).

\section{DISCUSSION}

Identifying molecular changes leading to BOS is essential for understanding disease development. We mapped total collagen, col IV and biglycan in transbronchial biopsies and related the results to later development of BOS and clinical parameters. We identified a locationspecific and time-specific pattern of these ECM proteins. To the best of our knowledge, this is the first report identifying early ECM changes associated with later BOS development. The findings do not yet allow suggestion of any causal mechanism.

The main findings of our study were: (1) alveolar and small airway compartments displayed different post-ltx changes; (2) patients with BOS showed different post-ltx changes compared with patients without BOS; (3) patients with early-onset BOS showed increased BOS severity and distinct, early ECM changes; and (4) early acute rejection episodes coincided with early ECM changes in patients developing BOS.

\section{Localisation of ECIM changes in distinct compartments}

Between 3 and 12 months post-ltx, we observed changes in the content of total collagen and col IV in the 

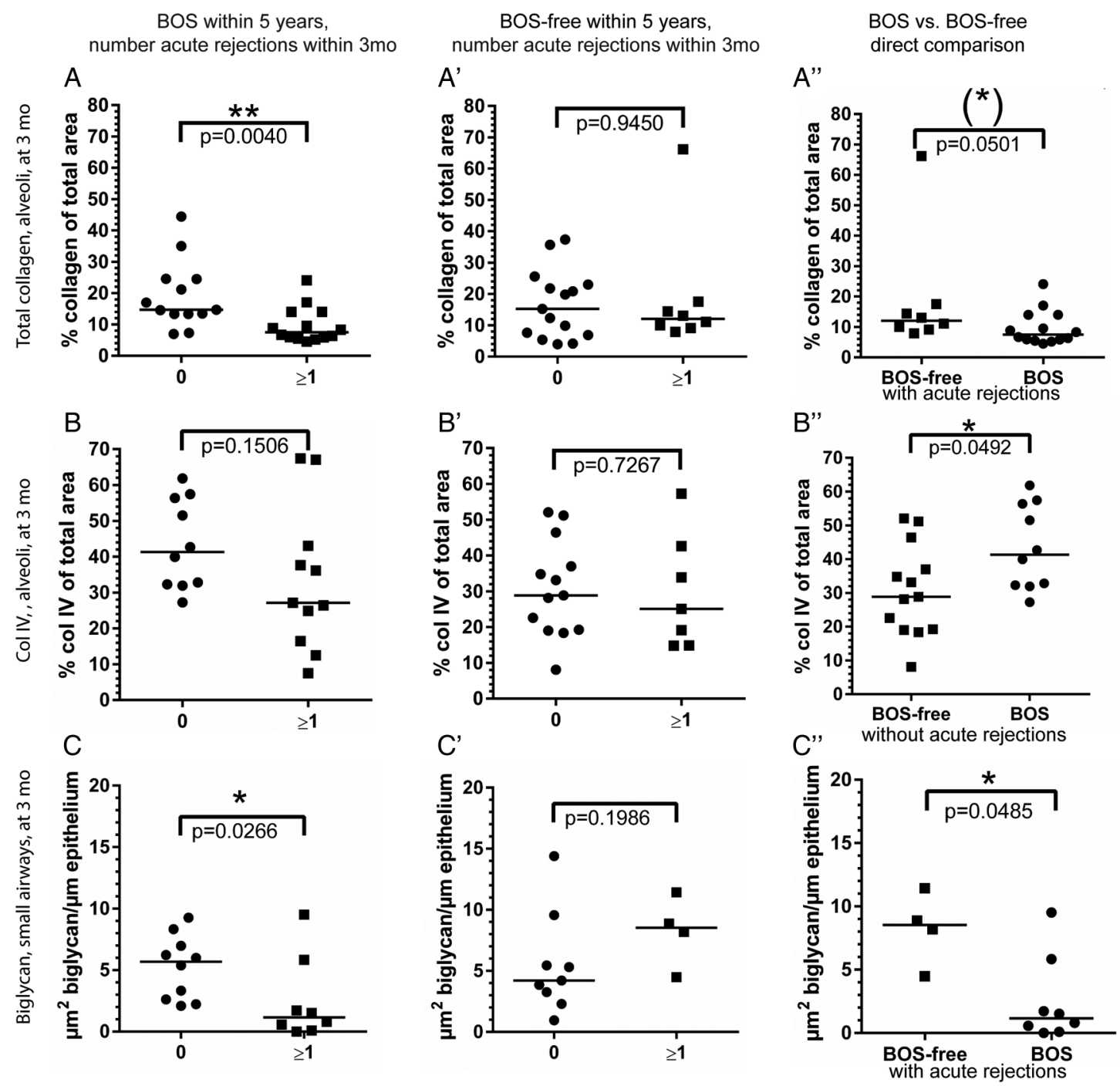

Figure 4 Treated acute rejection and ECM changes at 3 months in patients with/without BOS. Depicted are changes in total alveolar collagen (A, $\left.A^{\prime}\right)$, alveolar col IV (B, B') and small airway biglycan $\left(C, C^{\prime}\right)$ at 3 months when comparing patients with/ without BOS who did or did not experience treated acute rejections within 3 months post-Itx. (A", B" and C") Direct comparisons between patients developing BOS and patients without BOS at 3 months. Scatter dot plots are shown with median values. Data were analysed using the Mann-Whitney test. BOS, bronchiolitis obliterans syndrome; col IV, collagen type IV; ECM, extracellular matrix; Itx, lung transplantation.

alveolar, but not the small airway, compartment. In contrast, biglycan changed in the small airways, but not in the alveoli. This pattern was consistent, both when analysing the whole patient set and when relating the data to subgroups according to later BOS status and occurrence of acute rejection (figures 1-4). This is interesting since changes in the small airways are well described in BOS while alveolar changes are less recognised due to inconspicuousness in routine histopathological assessments. ${ }^{3} 1314$ General changes, irrespective of BOS, have been described before, probably being part of an adaptation response. ${ }^{1322} 25$

We also observed differences within the ECM. The BM is a thin, sheet-like specialisation of the ECM. Col IV, a BM marker, was found to increase in the alveoli between 3 and 12 months in patients who remained BOS-free.
Total collagen and biglycan, proteins of the interstitial ECM, increased in patients who developed BOS. Summarising, our data argue for distinct post-ltx mechanisms in different parts of the transplanted lung, possibly playing a part in BOS pathogenesis.

\section{The time aspect in post-Itx changes of the ECIM and development of BOS}

We did not find a difference at 3 or 12 months in the content of total collagen, col IV or biglycan when directly comparing all patients who did, or did not, develop BOS. However, differences became apparent when focusing on changes between 3 and 12 months (figure 2). This underlines the importance of the time aspect when investigating BOS as well as the value of paired data. The data presented for total collagen and col IV were analysed 
using the Wilcoxon matched-pairs test when applicable. Owing to a lack of paired data, this was not possible for biglycan and the Mann-Whitney test was used. However, we find the data convincing when plotting the individual differences (figure 2C").

The time factor is also illustrated by the known heterogeneity in onset and disease progression of patients with BOS, ${ }^{6} 2627$ which we also see in our patient set (table 1). Patients with early-onset BOS and with a high BOS grade displayed distinct ECM changes (figure 3).

The goal of studies like ours is to identify predictive markers. Interestingly, patients with future BOS displayed decreased alveolar total collagen and small airway biglycan at 3 months when having experienced treated acute rejections, compared with patients with BOS who did not have these (figure 4). This relation could not be seen for patients without BOS.

These post-ltx changes are remarkable when considering the crucial effect of the ECM on lung architecture and cellular behaviour. ${ }^{28}$ Collagens are low-turnover proteins but their deposition can change with appropriate stimuli as observed in wound healing. ${ }^{29}{ }^{30}$ Proteoglycans like biglycan can react to changes in the microenvironment, for example, by being cleaved and releasing fibrotic mediators or by affecting cell migration. ${ }^{20} 3132$ Granzyme B, an inflammatory serine protease whose expression increases in BAL during acute rejection, cleaves biglycan, thereby releasing active TGF- $\beta 1$, a major inducer of fibrotic processes. ${ }^{15} 334$

Our data on ECM protein changes after ltx are in good agreement with the current hypothesis of BOS development. ${ }^{7}$ Early post-ltx events, for example, during acute rejection, lead to tissue damage that might be illustrated by the decrease in ECM proteins at 3 months in patients with BOS with acute rejections. This is counteracted by repair mechanisms leading to ECM deposition as seen in our study by an increase in total alveolar collagen and small airway biglycan between 3 and 12 months in patients with future BOS. Finally, this process might become uncontrolled, possibly leading to fibrosis.

The mechanistic link between pre-ltx, peri-ltx and post-ltx events; the occurrence of acute rejection episodes; and the development of chronic rejection is until now not established. While there are a multitude of studies examining cellular immunological events, including both the innate and the adaptive immune system, data conclusively describing the effect on the ECM molecules are still scarce. ${ }^{35} 36$ We speculate that the massive influx of immune cells during acute rejection, secreting various cytokines, creates a catabolic environment, most likely by production and activation of matrix metalloproteinases (MMP). Elevated levels of MMP-8 and MMP-9, proteases degrading collagens, as well as proteoglycans like biglycan, have been shown to precede BOS. ${ }^{37-39}$ These processes might be enhanced by a possible protein catabolic effect of methylprednisolone during treatment of acute rejection episodes. ${ }^{40}$ On a histological level, acute rejections resolve completely; consequently, a restoration of tissue homoeostasis must occur. Macrophages are cells which potentially might link together acute and chronic rejection. ${ }^{41}$ While activated M1-like macrophages are involved in acute tissue inflammation, there might be a polarisation towards M2-like macrophages in the later resolving phase. ${ }^{36} 4243$ Interestingly, certain M2 macrophage subtypes, most prominently M2c macrophages, have been implicated to actively take part in fibrotic processes. ${ }^{44}{ }^{45}$ However, more research effort is needed to understand processes leading to the development of CLAD.

Our study provides a proof of concept on how to follow post-transplantation events in the ECM on a molecular level. We suggest using this approach further in larger independent studies involving patient cohorts from different transplantation centres.

This study is based on small forceps biopsies, which increases the feasibility for all lung transplant centres to implement this technique, but limits conclusions one may draw on the whole lung. This bigger picture is the aim of studies visualising post-ltx changes in explants with advanced imaging techniques. ${ }^{46} 47$

Our study emphasises the importance of ECM changes possibly leading to BOS, where time is an important factor as well as the localisation to distinct compartments of the transplanted lung.

\section{Author affiliations}

${ }^{1}$ Lung Biology Unit, Department of Experimental Medical Science, Lund University, Lund, Sweden

${ }^{2}$ Section for Lung Transplantation, Department of Cardiology, Copenhagen University Hospital, Rigshospitalet, Copenhagen, Denmark

${ }^{3}$ Department of Respiratory Medicine and Allergology, Lund University Hospital, Lund, Sweden

${ }^{4}$ Department of Pathology, Copenhagen University Hospital, Rigshospitalet, Copenhagen, Denmark

Acknowledgements The authors would like to thank Maria Weitoft for technical assistance with the Visiomorph programme and Ulrika Blank Savukinas for proofreading.

Contributors CM wrote the manuscript, performed immunohistochemistry, analysed and quantified the outcome of the $\mathrm{IHC}$ and histological stainings and interpreted them in relation to clinical data. HHS, CBA and MI provided clinical expertise, patient material and clinical data. CBA graded the patient material with regard to acute and chronic rejection and provided Masson's Trichrome stained tissue sections. AA-S and LTE provided clinical expertise and contributed to the interpretation of the data. AA-S, MI and GW-T designed the overall concept of the study. All authors participated in the study design, contributed to all manuscript versions and approved the final version of the manuscript.

Funding This study was supported by the Swedish Medical Research Council (11550), the Evy and Gunnar Sandberg Foundation, the Swedish Heart-Lung Foundation, the Alfred Österlund Foundation, the Royal Physiographic Society in Lund, the Swedish Foundation for Strategic Research, ALF (Government Public Health Grant) and the Medical Faculty of Lund University. HHS received a research grant from the Research Council at The Heart Centre, Copenhagen University Hospital, Rigshospitalet, Denmark and from Scandiatransplant.

Competing interests None declared.

Ethics approval The Danish Data Protection Agency.

Provenance and peer review Not commissioned; externally peer reviewed.

Data sharing statement No additional data are available. 
Open Access This is an Open Access article distributed in accordance with the Creative Commons Attribution Non Commercial (CC BY-NC 4.0) license, which permits others to distribute, remix, adapt, build upon this work noncommercially, and license their derivative works on different terms, provided the original work is properly cited and the use is non-commercial. See: http:// creativecommons.org/licenses/by-nc/4.0/

\section{REFERENCES}

1. Yeung JC, Keshavjee S. Overview of clinical lung transplantation. Cold Spring Harb Perspect Med 2014;4:a015628.

2. Sato $M$. Chronic lung allograft dysfunction after lung transplantation: the moving target. Gen Thorac Cardiovasc Surg 2013;61:67-78.

3. Stewart S, Fishbein MC, Snell Gl, et al. Revision of the 1996 working formulation for the standardization of nomenclature in the diagnosis of lung rejection. J Heart Lung Transplant 2007;26:1229-42.

4. Verleden GM, Raghu G, Meyer KC, et al. A new classification system for chronic lung allograft dysfunction. $J$ Heart Lung Transplant 2014;33:127-33.

5. Sato M, Waddell TK, Wagnetz U, et al. Restrictive allograft syndrome (RAS): a novel form of chronic lung allograft dysfunction. $J$ Heart Lung Transplant 2011;30:735-42.

6. Meyer KC, Raghu G, Verleden GM, et al. An international ISHLT/ ATS/ERS clinical practice guideline: diagnosis and management of bronchiolitis obliterans syndrome. Eur Respir J 2014;44:1479-503.

7. Belperio JA, Weigt SS, Fishbein MC, et al. Chronic lung allograft rejection: mechanisms and therapy. Proc Am Thorac Soc 2009;6:108-21.

8. Burton $\mathrm{CM}$, Iversen $\mathrm{M}$, Carlsen $\mathrm{J}$, et al. Acute cellular rejection is a risk factor for bronchiolitis obliterans syndrome independent of post-transplant baseline FEV1. J Heart Lung Transplant 2009;28:888-93.

9. Kennedy VE, Todd JL, Palmer SM. Bronchoalveolar lavage as a tool to predict, diagnose and understand bronchiolitis obliterans syndrome. Am J Transplant 2013;13:552-61.

10. Fisichella PM, Davis CS, Lowery E, et al. Aspiration, localized pulmonary inflammation, and predictors of early-onset bronchiolitis obliterans syndrome after lung transplantation. J Am Coll Surg 2013;217:90-100. Discussion 00-1.

11. Jonigk $\mathrm{D}$, Izykowski $\mathrm{N}$, Rische $\mathrm{J}$, et al. Molecular profiling in lung biopsies of human pulmonary allografts to predict chronic lung allograft dysfunction. Am J Pathol 2015;185:3178-88.

12. Krustrup $D$, Iversen $M$, Martinussen $T$, et al. Time elapsed after transplantation influences the relationship between the number of regulatory $T$ cells in lung allograft biopsies and subsequent acute rejection episodes. Transpl Immunol 2014;31:42-7.

13. Andersson-Sjöland A, Erjefält JS, Bjermer L, et al. Fibrocytes are associated with vascular and parenchymal remodelling in patients with obliterative bronchiolitis. Respir Res 2009;10:103.

14. Hirabayashi T, Demertzis S, Schäfers J, et al. Chronic rejection in lung allografts: immunohistological analysis of fibrogenesis. Transpl Int 1996;9(Suppl 1):S293-5.

15. Wynn TA, Ramalingam TR. Mechanisms of fibrosis: therapeutic translation for fibrotic disease. Nat Med 2012;18:1028-40.

16. Maina JN, West JB. Thin and strong! The bioengineering dilemma in the structural and functional design of the blood-gas barrier. Physiol Rev 2005;85:811-44.

17. West JB, Mathieu-Costello O. Structure, strength, failure, and remodeling of the pulmonary blood-gas barrier. Annu Rev Physiol 1999;61:543-72.

18. Khoshnoodi J, Pedchenko V, Hudson BG. Mammalian collagen IV. Microsc Res Tech 2008;71:357-70.

19. Hardingham TE, Fosang AJ. Proteoglycans: many forms and many functions. FASEB J 1992;6:861-70.

20. Nastase MV, lozzo RV, Schaefer L. Key roles for the small leucine-rich proteoglycans in renal and pulmonary pathophysiology. Biochim Biophys Acta 2014;1840:2460-70.

21. Weitoft M, Andersson C, Andersson-Sjöland A, et al. Controlled and uncontrolled asthma display distinct alveolar tissue matrix compositions. Respir Res 2014;15:67.
22. Andersson-Sjöland A, Thiman L, Nihlberg K, et al. Fibroblast phenotypes and their activity are changed in the wound healing process after lung transplantation. $J$ Heart Lung Transplant 2011;30:945-54.

23. Burton CM, Carlsen J, Mortensen J, et al. Long-term survival after lung transplantation depends on development and severity of bronchiolitis obliterans syndrome. J Heart Lung Transplant 2007;26:681-6.

24. Wilusz RE, Sanchez-Adams J, Guilak F. The structure and function of the pericellular matrix of articular cartilage. Matrix Biol 2014;39:25-32.

25. Law L, Zheng L, Orsida B, et al. Early changes in basement membrane thickness in airway walls post-lung transplantation. $J$ Heart Lung Transplant 2005;24:1571-6.

26. Lama VN, Murray S, Lonigro RJ, et al. Course of FEV(1) after onset of bronchiolitis obliterans syndrome in lung transplant recipients. $A m$ $J$ Respir Crit Care Med 2007;175:1192-8.

27. Jackson $\mathrm{CH}$, Sharples LD, McNeil K, et al. Acute and chronic onset of bronchiolitis obliterans syndrome (BOS): are they different entities? J Heart Lung Transplant 2002;21:658-66.

28. Davidson JM. Biochemistry and turnover of lung interstitium. Eur Respir J 1990;3:1048-63.

29. Maquart FX, Monboisse JC. Extracellular matrix and wound healing Pathol Biol (Paris) 2014;62:91-5.

30. Rucklidge GJ, Milne G, McGaw BA, et al. Turnover rates of different collagen types measured by isotope ratio mass spectrometry. Biochim Biophys Acta 1992;1156:57-61.

31. Nastase MV, Young MF, Schaefer L. Biglycan: a multivalent proteoglycan providing structure and signals. J Histochem Cytochem 2012;60:963-75.

32. Tufvesson E, Westergren-Thorsson G. Biglycan and decorin induce morphological and cytoskeletal changes involving signalling by the small GTPases RhoA and Rac1 resulting in lung fibroblast migration. $J$ Cell Sci 2003;116(Pt 23):4857-64

33. Madsen $C B$, Norgaard $A$, Iversen $M$, et al. Elevated mRNA levels of CTLA-4, FoxP3, and granzyme B in BAL, but not in blood, during acute rejection of lung allografts. Transpl Immunol 2010;24:26-32

34. Boivin WA, Shackleford M, Vanden Hoek A, et al. Granzyme B cleaves decorin, biglycan and soluble betaglycan, releasing active transforming growth factor-beta1. PLOS ONE 2012;7:e33163.

35. Ingulli $E$. Mechanism of cellular rejection in transplantation. Pediatr Nephrol 2010;25:61-74.

36. Mori DN, Kreisel D, Fullerton JN, et al. Inflammatory triggers of acute rejection of organ allografts. Immunol Rev 2014;258:132-44.

37. Genovese F, Barascuk N, Larsen L, et al. Biglycan fragmentation in pathologies associated with extracellular matrix remodeling by matrix metalloproteinases. Fibrogenesis Tissue Repair 2013;6:9.

38. Smith GN Jr, Mickler EA, Payne KK, et al. Lung transplant metalloproteinase levels are elevated prior to bronchiolitis obliterans syndrome. Am J Transplant 2007;7:1856-61.

39. Babu AN, Nicolls MR. The matrix comes to lung transplantation. Transplantation 2007:83:683-4.

40. Schakman O, Gilson H, Thissen JP. Mechanisms of glucocorticoid-induced myopathy. J Endocrinol 2008;197:1-10.

41. Jiang $X$, Tian $W$, Sung $Y K$, et al. Macrophages in solid organ transplantation. Vasc Cell 2014;6:5.

42. Stables MJ, Shah S, Camon EB, et al. Transcriptomic analyses of murine resolution-phase macrophages. Blood 2011;118:e192-208.

43. Lech M, Anders HJ. Macrophages and fibrosis: How resident and infiltrating mononuclear phagocytes orchestrate all phases of tissue injury and repair. Biochim Biophys Acta 2013;1832:989-97.

44. Martinez FO, Gordon S. The M1 and M2 paradigm of macrophage activation: time for reassessment. F1000Prime Rep 2014;6:13.

45. Chang MY, Chan CK, Braun KR, et al. Monocyte-to-macrophage differentiation: synthesis and secretion of a complex extracellular matrix. J Biol Chem 2012:287:14122-35.

46. Verleden SE, Vasilescu DM, McDonough JE, et al. Linking clinical phenotypes of chronic lung allograft dysfunction to changes in lung structure. Eur Respir J 2015;46:1430-9.

47. Verleden SE, Vasilescu DM, Willems $\mathrm{S}$, et al. The site and nature of airway obstruction after lung transplantation. Am J Respir Crit Care Med 2014;189:292-300. 